\title{
Characterization of measured lightning electric fields observed in Malaysia
}

\begin{abstract}
This paper presents a characteristic of lightning electric fields. The lightning electric fields captured by two parallel plats sensor and the characterization for preliminary breakdown pulse (PBP) and first return stroke (RS) were studied. A total of 207 data were recorded and 10 data are selected and been utilized in this paper accordingly. In PBP signal data, time before return stroke, ending time and maximum peak of PBP were analyzed. The time duration between the PBP and the occurrence of RS was obtained as $0.64 \mathrm{~ms}$ and $2.61 \mathrm{~ms}$ and then compared with results from other researchers. For RS signal data, slow front time, deviation time, RS peak, time to peak, zero crossing time and $10 \%$ to $90 \%$ rise time were evaluated meticulously and the results were discussed accordingly. Moreover, the statistical correlation between electric field zero times and corresponding rise times was studied.
\end{abstract}

Keyword: Characteristic; Electric field; Lightning; Return stroke 